\title{
Mitotic Index In Invasive Ductal Carcinoma Nst (IDC-Nst) of Breast and Its Relation With Tumor Size and Regional Lymph Nodes Status
}

\author{
Setyaningrum DAW \\ Departement of Anatomical Pathology \\ Faculty of Medicine, Trisakti University \\ Jakarta, Indonesia
}

\author{
Siregar NC, Tjahjadi $H$ \\ Departement of Anatomical Pathology \\ Faculty of Medicine, University of Indonesia \\ Jakarta, Indonesia
}

\begin{abstract}
Until nowadays, breast cancer is still the highest malignancy in women in both the developing and developed countries. The main causes of death in breast cancer is metastasis. Patients who have experienced distant metastases have a poor prognosis. Proliferation is a biological process that is important for its role in growth and in maintaining tissue homeostasis, so that cell proliferation assessment is often used to predict the behavior of tumors, among others, the potential for recurrence, metastasis potential, to the ability to survive. Plays an important role in the proliferation of invasive breast cancer. Increased proliferation strongly linked with a poor prognosis. This is because an increase in proliferation significant increase in the aggressiveness of the tumor. Mitotic index is one of proliferation assessment that used in breast cancer histopathological staging. Objective: to know the relationship between mitotic index, tumor size, regional lymph node involvement, in invasive ductal breast carcinoma NST. The 47 paraffin block preparations of invasive ductal breast carcinoma NST obtained from the Jakarta Breast Center the period 20082011 conducted hematoxylin eosin staining (HE) to calculate mitotic index. Secondary data such as age, histopathological diagnosis and grading, tumor diameter, and the involvement of lymph nodes obtained from medical record of histopathological examination result sheets. Analysis with Chi-square test and Spearman. There is no correlation between mitotic index, tumor size, regional lymph node involvement on invasive ductal carcinoma (IDC-NST) of breast in this study.
\end{abstract}

Keywords: mitotic index; tumor size; regional lymph node involvement; invasive ductal carcinoma NST of breast

\section{INTRODUCTION}

Until now, breast cancer is still the most common malignancy in women in both developing and developed countries. From the IARC and WHO data show that in 2008 breast cancer patients reached 1.38 million new cases in the world which means $23 \%$ of all cancers and ranked second [1]. Data from the Cancer Registry in Indonesia shows that in 2009 breast cancer became most malignancies in women in Indonesia. In the United States, breast cancer remains the most common invasive cancer in women and is the second leading cause of cancer death; it is estimated that breast cancer claimed the lives of more than 39,000 people both men and women in 2012, with more than 229,000 new diagnoses the same year. Worldwide, it is estimated that 1.4 million women a year receive a diagnosis of breast cancer [3]. In China, as in most other countries, breast cancer is now the most common cancer in Chinese women; cases in China account for $12.2 \%$ of all newly diagnosed breast cancers and $9.6 \%$ of all deaths from breast cancer worldwide [4].

It is estimated that $7-10 \%$ of breast cancer patients are diagnosed with distant metastatic, and $30 \%$ of patients who are not metastasized initially end up with metastasis. The main cause of death in breast cancer is the presence of metastasis. Patients with distant metastasis have a poor prognosis with an estimated 5-year survival rate of only $21 \%$ [5]. Regional dispersal of lymph nodes can predict distant metastases but in $20-30 \%$ of patients with distant metastases no spread of lymph nodes is present. This condition indicates that distant spread may occur directly to the blood vessels without passing through the lymph nodes [6].

The prognosis of invasive breast carcinoma is determined by various factors both clinically and histopathologically. First category prognostic factors include tumor size, lymph node involvement status, micrometastasis, histopathologic type, mitotic index, and ER/PR status [7].

The number of mitosis is one of the factors in the determination of histopathologic grading of invasive breast carcinoma in addition to the formation of tubules/glands and nuclei pleomorphism [1].

The mitotic index is also used by clinicians to determine the treatment protocol. In invasive breast cancer patients without lymph node involvement, with a tumor size between $1-3 \mathrm{~cm}$ and mitotic index (MAI = mitotic activity index $)<10 / 1.7 \mathrm{~mm} 2$ then no adjuvant therapy, whereas patients with MAI $\geq 10 / 1,7 \mathrm{~mm} 2$ should be given chemotherapy and/or additional hormonal therapy depending on the status of its steroid hormone receptors [8].

The importance of examining mitotic index in breast malignancy is the reason for the need to see whether there is a relationship between mitosis and tumor size and lymph node involvement in IDC NST breast escpecially in Indonesia. 


\section{Methodology}

There are fifty sample of this cross sectional study that obtained by tracing medical record data of patients diagnosed with invasive ductal carcinoma NST at Jakarta Breast Center (JBC) from 2008 - 2011, collected their FFPE for microscopic preparation with $\mathrm{HE}$ staining. All of the samples were reconfirmed by pathologist as invasive breast carcinomas of NST.

The research is mainly carried out in the Histopathology Laboratory of Faculty of Medicine University of Indonesia in Jakarta.

The samples used in this study were formalin-fixed paraffin embedded (FFPE) tissue of invasive breast carcinoma NST obtained from Jakarta Breast Center period 2008 - 2011. Secondary data of age, histopathologic diagnosis, grading, size of tumor, and lymph node status were obtained from medical record of histopathological examination result sheets.

\section{A. Hematoxylin Eosin (HE) Staining Method}

The paraffin embedded breast cancer tissue is cut with microtom at 4-5 $\mu \mathrm{m}$ thickness. The slice is inserted into the waterbath, taken with a glass object and dried in hotplate for further HE staining process. Followed by washing the paraffin with xylol, and series of alcohol $100 \%, 90 \%, 80 \%$, and $70 \%$.

Next step is soaked in Hematoxylin solution, wash under running water then examined under a microscope. If the color is too blue then the preparation was washed back under running water until it reaches a pretty good blue color. Next counterstaining with Eosin dye solution. Dehydration was done alcohol serial starts from 70\%, 80\%, 90\%, and $100 \%$. Last step was clarified in xylol.

\section{B. Mitotic Index Scoring Method}

The number of mitoses is counted from the number of mitotic images using a light microscope at 40x objective lens (high power field/hpf) in 10 random field of view. If a mitotic image is found in the form of two parallel chromosome spindles is calculated as two mitosis. ${ }^{1}$ The mitotic index is divided into two groups: $\leq 9 / 10 \mathrm{hpf}$, and $\geq 10 / 10 \mathrm{hpf}$.

Selected field for mitotic scoring should be from the peripheral leading edge of the tumour. If there is heterogeneity, regions exhibiting a higher frequency of mitoses should be chosen. ${ }^{1}$ Mitosis is calculated in the infiltrating area and avoided in situ component, in areas that proliferate actively that found many cells in their mitotic phase, without necrosis, inflammation or calcification. ${ }^{9}$ Mitotic features appear as thick, hyperchromatic chromosomal clumps, without nuclear envelope and basophilic cytoplasm. ${ }^{8,10}$ Hyperchromatic and pyknotic nuclei are ignored since they are more likely to represent apoptosis than proliferation. ${ }^{1}$

The tumor size was taken from the largest dimension of tumor that written in medical record sheets than divided into two groups: tumor size $<2 \mathrm{~cm}$ (T1) and $>2 \mathrm{~cm}$ (> T2) according to the classification of histopathologic staging based on tumor size.
Analysis of mitotic index, tumor diameter size, lymph node status using Chi-Square or Fisher's Exact or KolmogorovSmirnov test. While correlation between variables done by Spearman correlation test. Further data will be presented in the form of narration, images, and tables.

\section{RESULTS}

From 50 samples collected there were 3 samples were removed because age and tumor size data were not found, so the total sample processed was 47 .

When performed a correlative hypothesis analysis of the relationship between age with tumor size and lymph node involvement with Spearman correlative test and the result is no correlation found $(\mathrm{p}>0,05)$.

Fig. 1 is microscopic images of invasive ductal carcinoma NST of breast with HE staining in 10x and 40x objective lens magnifications and an overview of the atypical mitosis seen in the preparation.

TABLE I. SAMPLE PROFILE

\begin{tabular}{|c|l|}
\hline \multicolumn{1}{|l|}{ Clinical data } & Description \\
\hline Age & $31-72$ y.o (mean 51 y.o) \\
\hline$<50$ y.o & $24(51.1 \%)$ \\
$\geq 50$ y.o & $23(48.9 \%)$ \\
unknown & $1(2 \%)$ \\
\hline Tumor size & \\
\hline$\leq 2 \mathrm{~cm}(\mathrm{~T} 1)$ & $15(31.9 \%)$ \\
$>2-5 \mathrm{~cm}(\mathrm{~T} 2)$ & $30(63.8 \%)$ \\
$>5 \mathrm{~cm}(\mathrm{~T} 3)$ & $2(4.3 \%)$ \\
\hline Lymph node status & \\
\hline N0 & $23(48.9 \%)$ \\
\hline N1 & $24(51.1 \%)$ \\
\hline
\end{tabular}

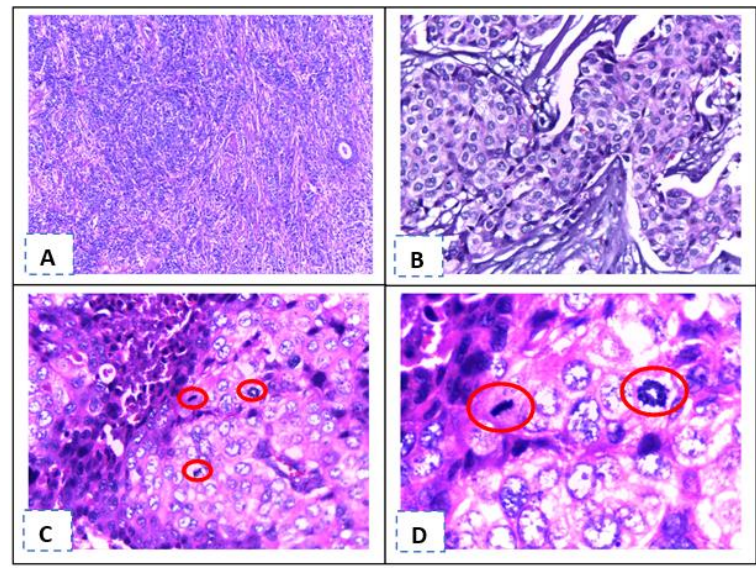

Fig. 1. Invasive ductal carcinoma NST of breast with HE staining. A. 10x objective lens magnification. B. 40x objective lens magnification. C. 40x objective lens magnification; D. 100x objective lens magnification in red circles. 
Then performed a correlative hypothesis analysis of the relationship between mitotis index with tumor size and lymph node involvement with Spearman correlative test and the result shows in table 2 below.

TABLE II. REFERENCES CORRELATION BETWEEN Mitosis INDEX WitH TUMOR SIZE, AND LYMPH NODE STATUS

\begin{tabular}{|c|c|c|}
\hline Variables & p vaolue & Interpretation \\
\hline Tumor size & 0,536 & $\mathrm{p}>0,05$ not significant \\
\hline Lymph node status & 0,434 & $\mathrm{p}>0,05$ not significant \\
\hline
\end{tabular}

\section{DISCUSSION}

The subjects were divided into two groups according to age, with consideration of some previous studies indicating an increased incidence of breast cancer at age 35-39 years and the peak is 50 years old. So in this study age group is divided into $<50$ years and $>50$ years. Table 1 showed that ther is a slightly higher incidence in younger age group $<50$ years old $(51.1 \%)$ compared to age group $>50$ years old $(48.9 \%)$.

When compared with research conducted at RSUP Cipto Mangunkusumo and National Cancer Center Dharmais in 2008 showed a tendency incidence of breast cancer patients increased at a young age [11].

This condition is also corroborated by epidemiological data from Population-Based Cancer Registries (PBCR) which states that the characteristics of breast cancer patients in Asian countries have been identified at a relatively young age [2].

The striking difference is that the peak age for breast cancer is between 40 and 50 years in the Asian countries, whereas the peak age in the Western countries is between 60 and 70 years. Also, the incidence of breast cancer in Asia is rising and is associated with increased mortality. The differences among the Asian countries need further studies regarding diagnosis, screening activities, lifestyle, genetic susceptibility [2].

From the correlative analysis did not show any significant correlation between mitotic index with tumor size and lymph node status. Theoretically, an increase in mitosis means an increase in tumor proliferation, which is a biologically important process for the tumor in maintaining its presence. Increasing proliferation means enlarging the tumor size but it turns out that in this study, there is no relationship between the index mitosis with tumor size.

Tumor size among others influenced by angiogenesis and tumor cell proliferation rate. However, it should be noted that there are other factors that play a role in cell proliferation include hormon and hormonal receptor (ER / PR) factors, as well as genetic and even possibility epigenetics that also play a role in the proliferation process. The effects of previous chemotherapy drugs that may have been given to subject in this study but remembering that the researchers did not collect data on previous therapeutic history, it still needs to be explored further on this possibility.

The results of this study indicate no correlation between mitotic index with lymph node involvement. This suggests other factors to consider that may affect metastasis to lymph nodes in invasive ductal carcinoma NST of breast tissue in this study include enzymes that work in the degradation of extracellular matrix which became the early step of metastasis such as matrix metalloproteins (MMPs) or their inhibitors, a system of urokinase-type plasminogen activator (uPA), or any other factors such as HIF-1, VEGF that have not been examined.

Many studies conducted over the last 2 decades on metastasis in carcinogenesis show the important role of MMPs. Some studies suggests that certain MMPs may also play a role in breast cancer initiation and growth other than oncogenes such as c-erbB-2, c-myc, ras, and tumour suppressor genes such as p53 and $\mathrm{Rb}$ that has become generally believed.

Indeed some of the c-oncogenes may contribute to tumourigenesis by regulating the expression of MMPs. For example, transfection of MCF-10A breast cancer cells with either c-erbB-2 or c-ras resulted in increased expression of MMP-2, whereas transfection of MCF-7 cells with the ets gene, PEA-3, led to increased production of MMP-9 [12].

Cancer originating from epithelial tissues such as invasive ductal dermal carcinoma, indicates the degree of malignancy based on its ability to invade and distant metastases. These tumor cells undergo changes in their development both in their form and in the attachment of the cells as well as in the extracellular matrix. This change is due to the disruption of cell adhesion molecules (CAMs) such as integrin and Ecadherin that play a role in adhesion between cells. On the other hand, molecules that play a role in cell migration when embryogenesis and inflammatory processes actually increase $[13,14]$.

These changes make it easier for tumor cells to invade the surrounding tissue and then penetrate into the blood vessels and or the nearest lymph vessels so that cancer cells enter the blood and lymphatic circulatory system and lymphogenic and hematogenous metastases. Further extravasation occurred, then formed a small nodule containing tumor cells (micrometastasis), and eventually micrometastasis developed into a macroscopic tumor (colonization) [14].

There is a hypothesis that breast cancer metastases are preceded by micrometastasis in dormant conditions. Micrometastasis is a metastasis seen in microscopic observations of $<2 \mathrm{~mm}$. In this hypothesis the micrometastasis occurs in the pre-clinical phase and does not experience growth or remain dormant until angiogenesis occurs. This process involves several factors released by tumor cells, host stroma cells and infiltrating lymphocytes. Angiogenesis causes increased proliferation of tumor cells so that tumors continue to grow [15].

Metastasis can even occur for years after the primary tumor is lifted. This is because tumor cells may spread during the early stages of growth and survive as dormant tumor cells or as far as micrometastasis until certain genetic or epigenetic changes have resulted in the tumor growth resulting in macrometastasis [12]. 


\section{CONCLUSION}

Many studies prove the proliferation rate associated with tumor size and even distant metastasis. Unfortunately, there is not enough evidence that mitotic index has correlation with tumor size and regional lymph node involvement on Invasive Ductal Carcinoma NST of breast in this study.

However, this is not the end result, it is still necessary to examine the proliferation with several different cell proliferation screening methods, as well as to examine the status of MMPs, hormonal status and other growth factors both proven and to be expected contributing to this malignancy in Indonesia.

\section{REFFERENCES}

[1] WHO. WHO classification of tumours of the breasts. IARC Press. 2012

[2] Curado MP. Breast cancer in the world: incidence and mortality. SaludPublica de Mexico. 2011; 53:372-384.

[3] Ban KA, Godellas CV. Epidemiology of breast cancer. Surg Oncol Clin N Am 23 (2014) 409-422. http://dx.doi.org/10.1016/j.soc.2014.03.011.

[4] Fan L, Weippl KS, Li JJ, St Louis J, Finkelstein DM, Yu KD, et al. Breast cancer in China. The Lancet. Oncology, 2014; 15 (7):e279-89.

[5] Bertucci F, Bimbaum D. Distant metastasis: not out of reach anymore. (minireview) J.Biol. 2009; 8:28

[6] Pantel K, Brakenhoff RH. Dissecting the metastatic cascade (review). Nature. 2004;4:448-456.

[7] Mandard AM, Denoux Y, Herlin P, Duigou F, van de Vijver MJ, Clahsen PC, et al. Prognostic value of DNA cytometry in 281 premenopausal patients with lymph node negative breast carcinoma randomized in a control trial. Multivariate analysis with Ki-67 index, mitotic count and microvessel density. Cancer 2000;89:1748-57.

[8] Van Diest PJ, Brugal G, Baak JPA. Proliferation markers in tumour: interpretation and clinical value. J.Clin.Pathol. 1998;51:716-24.

[9] Medri L, Volpi A, Nanni O, Vecci AM, Mangia A, Schittulli F, et al. Prognostic relevance of mitotic activity in patients with node-negative breast cancer. Modern Pathology 2003;16:1067-1075.

[10] Van Diest PJ, Wall EVD, Baak JPA. Prognostic value of proliferation in invasive breast cancer: a review. J.ClinPathol. 2004;57:675-681.

[11] Sutandyo N, Suzanna E, Haryono SJ, Reksodiputro AH. Signaling pathways in early onset sporadic breast cancer of patients in Indonesia. Acta Med Indones-Indones J Intern Med. 2008; 40:139-145.

[12] Duffy MJ, Maguire TM, Hill A, McDermott E, O’Higgins N. Metalloproteinases: role in breast carcinogenesis, invasion and metastasis. Breast Cancer Res. 2000, 2:252-7.

[13] Beckmann MW, Niederacher D, Schnurch HG, Gusterson BA, Bender HG. Multistep carcinogenesis of breast cancer and tumour heterogeneity. (review) J Mol Med. 1997; 75:429-439.

[14] Hanahan D, Weinberg RA. Hallmarks of cancer: the next generation.(review). Cell. 2011 March 4;144:646-674.

[15] De More N, Van Zee KJ, Linkov I, BorgenPI, Gerald Wl.Biological behavior of human breast cancer micrometastasis, Clin Cancer Res. 2001;7:2434-2439. 